\title{
Root cause analysis of the folding deformation for the expansion joint in the nuclear power station
}

\author{
Li Nan, Che Yin hui, Li Guo dong, Zu Shuai and Guan Jian jun \\ SuZhou Nuclear Power research Institute, ShenZhen, 518038, China
}

\begin{abstract}
In the nuclear power station, the expansion joint of moisture separator re-heater (MSR for short) was founded locally folding deformation under the outage condition. Folding deformation increasing will lead expansion joint leak, it would cause emergency stop when leaking seriously. To solve these problems, this paper analysis mainly on three aspects: the material organization performance, the expansion joint installation and the stress characteristic of expansion joint. Both the verticality deviation in the process of installation and the material hardness value exceed standard are the root cause of these problems. At the end of the paper, the improvements have been put forward for this problem.
\end{abstract}

\section{Introduction}

The moisture separator re-heater (MSR for short) is set up between high pressure cylinder and low pressure cylinder in the Pressurized Water Reactor (PWR for short) nuclear power station. Its role is to separate new saturated steam from high pressure cylinder whose humidity is increasing after working. Then the new saturated steam go through two levels of re-heater, making the exhaust steam of high pressure cylinder become superheated steam after working and before entering the medium and low pressure cylinder. After through the separator, the water in steam has been wiped out, and it can reduce the water droplets on the turbine blade erosion, improve the efficiency of thermal system ${ }^{[1]}$.

The first and second level heating steam which is coming from the main steam system mostly condenses into water after finish heat-exchange in the re-heater tube, then going through the first and second level hydrophobic pipeline to the drain tank. The hydrophobic pipeline should be through the MSR shell, when the plant is running, the pipe material expanding or the stress appearing causes the horizontal displacement increases and the expansion amount of shell not synchronized. Therefore, set the expansion joints at the connection of drain pipe and shell to compensate thermal expanding displacement in every direction ${ }^{[2]}$.

This paper through metallographic analysis, mechanical property analysis and other analysis methods, on the basis of displacement measurement of expansion joint, establishes the expansion joint failure models of moisture separator re-heater, analyses the expansion joint stress, and explores failure causes of the MSR expansion joint in the nuclear power plant under the engineering application.

\section{Metallography analysis}

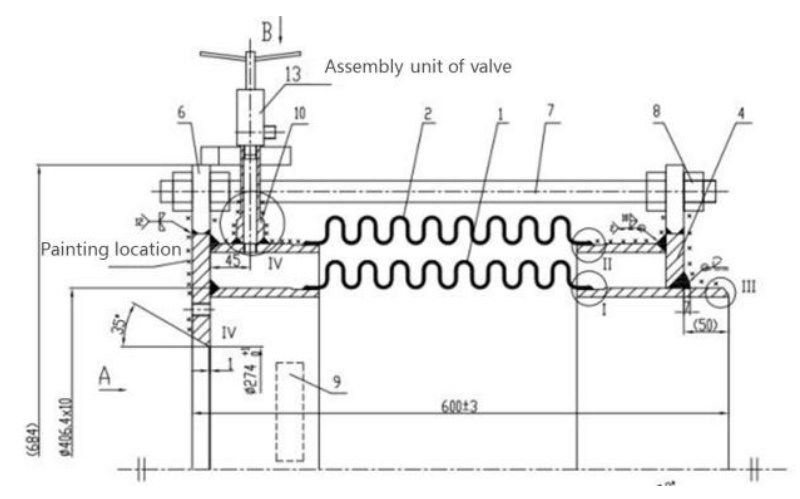

Figure 1. First and second level hydrophobic expansion joint.

Table 1. The equipment parameters of expansion joint.

\begin{tabular}{|l|l|}
\hline item & parameter \\
\hline Design pressure & $1.4 \mathrm{Mpa}$ \\
\hline $\begin{array}{l}\text { Design } \\
\text { temperature }\end{array}$ & $311^{\circ} \mathrm{C}$ \\
\hline $\begin{array}{l}\text { compensation } \\
\text { dosage }\end{array}$ & $\begin{array}{l}\text { axial direction is } 10 \mathrm{~mm} \text {, radial } \\
\text { direction is } 4 \mathrm{~mm}\end{array}$ \\
stiffness & $\begin{array}{l}\text { axial direction is } 1087 \mathrm{~N} / \mathrm{mm} \text {, radial } \\
\text { direction is } 441 \mathrm{~N} / \mathrm{mm}\end{array}$ \\
\hline Design fatigue life & $>1000$ \\
\hline $\begin{array}{l}\text { installation } \\
\text { characteristics }\end{array}$ & Welding \\
\hline
\end{tabular}

The folding deformation expansion joint is secondary hydrophobic expansion joint of MSR in this nuclear power station. Expansion joint has inside and outside part; each part divided into 5 layers and each layer is $0.6 \mathrm{~mm}$. 
Through hydraulic forming making the internal and external layers fit closely, as shown in figure 1 . According to maintenance manual operation, the equipment parameters of first and second level hydrophobic expansion joint are as in Table 1.

\subsection{Macroscopic observation analysis}

Three corrugated segments of expansion joint has folding deformation on the side where is taking over the end surface area. The maximum deformation position has about $20 \mathrm{~mm}$ length crevasse shape defect, it has obviously plastic deformation on both ends of the defect, as shown in figure 2. When cut the both ends of the expansion joint, found that deformation is only in the innermost layer 1 where is taking over the end surface area near the bottom part of the expansion joint, no deformation appears in the other four layers. The outside part expansion joint does not have deformation, the ends of the first layer and the rest four layers exists gap, which was not totally fit.

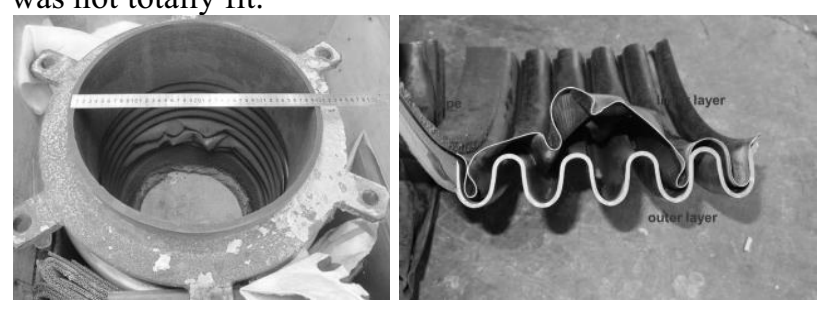

Figure 2. The deformation morphology of expansion joint.

Peeling off the innermost layer 1 where has folding deformation, found that the rest four layers of corrugated pipe have no crack, and no traces of corrosion around the crevasse. Using asana microscope observed inside and outside section morphology of the expansion joint, wave height of inside part is $29.6 \mathrm{~mm}$ by measured, pitch of wave is $41.6 \mathrm{~mm}$. The wave height of outside part is 29.1 $\mathrm{mm}$ by measured, pitch of wave is $36.2 \mathrm{~mm}$. The design of wave height is $29 \mathrm{~mm}$, pitch of wave is $36 \mathrm{~mm}$. Measurement data shows that the inside part of expansion joint has been out of shape.

\subsection{Chemical composition analysis}

Sampling for the innermost layer of expansion joint, using the PerkinElmer Optima 2100DV inductively coupled plasma emission spectrometer and element $\mathrm{C} 、 \mathrm{~S}$ analyzer for chemical composition analysis; the results are shown in table 2 .

Chemical composition results show that the chemical composition of innermost layer 1 has big differences with the product quality book which is provided by upstream suppliers. The content of $\mathrm{C}$ element beyond the specified value in the ASTM A240, other elements contents are in accordance with the ASTM A240 standard requirement for 304L stainless steel chemical composition ${ }^{[3]}$.

\subsection{Hardness analysis}

According to GB/T 4340.1-2009 'metal materials Vickers hardness test Part 1: test method'. Vickers hardness test was carried out on the sample; the results are shown in table 3 .

Vickers hardness test results show that the hardness values of sampling location are higher than ASTM A240 standard of 304L stainless steel. Hardness values exceeds the standard would make the material brittleness, and prone to cracking.

\subsection{Metallographic analysis}

The inner layer with and without deformation has been metallographic analysis, sampling inspection results show that the sampling position of microstructure are twin austenitic, grain size is class $7-8$. Organization is relatively uniform, and microstructure has no problem.

\section{Displacement detection analysis}

The calculation instruction shows that in order to meet the requirements of the fatigue life, the maximum horizontal displacement of the expansion joint when it is running should satisfy the following conditions: the horizontal displacement of operating equipment $(\leqslant 4 \mathrm{~mm})$ + horizontal displacement of installation $(\leqslant 5 \mathrm{~mm})=$ total horizontal displacement $(\leqslant 9 \mathrm{~mm})$, as shown in figure 3 . According to the calculation results, after the radial displacement changes into $9 \mathrm{~mm}$, the fatigue life significantly will reduce to 1115 times $(>1000)$ which is about $1 / 10$ of the initial design value $(4 \mathrm{~mm})^{[4]}$.

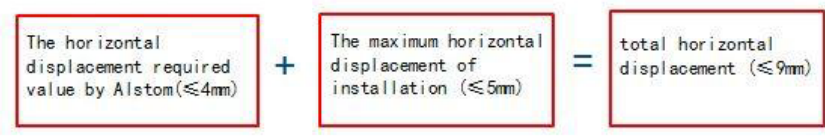

Figure 3. The displacement design requirements of expansion joint.

Analysed the reason of excessive displacement, finding the possible reasons mainly include the following two aspects:

(1) The assembling influence before installation: welding the expansion joint with MSR by poor control verticality, may cause the expansion joint deflect ${ }^{[2]}$.

Query unit 1 \# and 2 \# MSR's End of Manufacturing Report (short for EOMR), the EOMR shows that, after the assembling complete, the maximum horizontal displacement is $4 \mathrm{~mm}$. There is little amount of permissible error for on-site installation and adjustment is difficult when installing.

(2) The influence of installation: welding the expansion joint with the MSR's bottom drain pipe by poor control verticality, may cause the expansion joint deflect.

\section{The displacement measurement}

The expansion joint of unit 1 plant was measured under the thermal state. The measurement scheme: using the plumb line to measure, if the plumb line close to the top period of the expansion joint first. The bottom section of 
Table 2. Chemical composition test results

\begin{tabular}{|l|c|c|c|c|c|c|c|}
\hline \multirow{2}{*}{ item } & \multicolumn{6}{|c|}{ chemical composition wt\% } \\
\cline { 2 - 8 } & $\mathrm{C}$ & $\mathrm{Mn}$ & $\mathrm{P}$ & $\mathrm{S}$ & $\mathrm{Si}$ & $\mathrm{Cr}$ & $\mathrm{Ni}$ \\
\hline inner layer & 0.038 & 1.55 & 0.009 & 0.007 & 0.36 & 18.06 & 8.12 \\
\hline the quality of the product specification & 0.0154 & 1.09 & 0.023 & 0.001 & 0.55 & 18.4 & 9.2 \\
\hline ASTM A240 & $\leq 0.030$ & $\leq 2.00$ & $\leq 0.045$ & $\leq 0.030$ & $\leq 0.75$ & $18.0 \sim 20.0$ & $8.0 \sim 12.0$ \\
\hline
\end{tabular}

Table 3. Vickers hardness (HV1) test results.

\begin{tabular}{|c|l|c|c|c|c|c|}
\hline NO. & \multicolumn{1}{|c|}{ location } & \multicolumn{3}{c|}{ Vickers hardness /HV1 } & average value \\
\hline \multirow{2}{*}{$\begin{array}{c}\text { The inner layer without } \\
\text { deformation }\end{array}$} & cross section & 237.6 & 242.3 & 240.5 & 247.0 & 242 \\
\cline { 2 - 7 } & longitudinal section & 231.9 & 226.1 & 234.3 & 238.3 & 233 \\
\hline \multirow{2}{*}{$\begin{array}{c}\text { The inner layer with } \\
\text { deformation }\end{array}$} & cross section & 254.4 & 251.0 & 254.3 & 248.6 & 252 \\
\cline { 2 - 7 } & longitudinal section & 245.8 & 256.8 & 242.4 & 225.9 & 243 \\
\hline $\begin{array}{r}\text { ASTM A240 } \\
\text { GB/T 1172 }\end{array}$ & & & \multicolumn{3}{|c|}{$\begin{array}{c}\leq 201 \mathrm{HB} \\
(\leq 206 \mathrm{HV})\end{array}$} \\
\hline
\end{tabular}

the expansion joints deviate from the plumb line is negative. If near the bottom part first, the upper part deviation value is positive.

The unit condition is: the nuclear power is $80.7 \% \mathrm{Pn}$, electric power is $850 \mathrm{MWe}$, the measurement results are shown in table 4. Measurement results show that, when the nuclear power is $80.7 \% \mathrm{Pn}$, the expansion joint of the horizontal displacement has been reached or exceed the maximum requirements of design. Nuclear power can reach $100 \%$ Pn under normal conditions, and the horizontal displacement those are measured in the table 4 will be further increased.

Table 4. The horizontal displacement of unit 1.

\begin{tabular}{ccccc}
\hline NO. & Location & \multicolumn{2}{c}{ horizontal displacement } & $\begin{array}{l}\text { Horizontal } \\
\text { displacement } \\
\text { offset }\end{array}$ \\
\hline & 1 & 69 & 72 & -3 \\
1 top & bottom & 71 & -6 \\
& 2 & 66 & 40 & -5 \\
& 3 & 49 & 29 & 5 \\
& 4 & 38 & 36 & 9 \\
& 5 & 41 & 62 & 9 \\
\hline
\end{tabular}

\section{Stress simulation of displacement}

According to the material parameters of expansion joint and combining with the conditions of operation and installation of expansion joint, the innermost layer corrugated pipe caused by displacement stress simulation has been carried out on the finite element stress analysis. The original designed displacement value is $4 \mathrm{~mm}$ and the maximum allowable value is $9 \mathrm{~mm}$. These two situations are simulated to analysis the expansion joint's stress. The finite element calculation results are as follows:

(1) When the horizontal displacement is $4 \mathrm{~mm}$, the stress nephogram is shown in figure 4

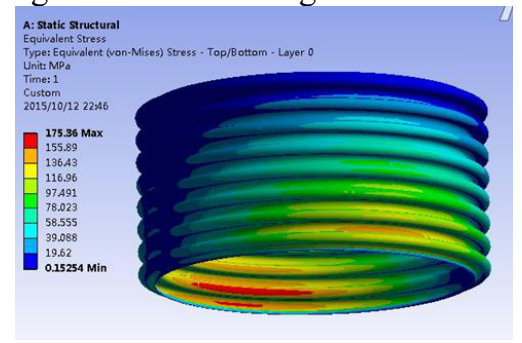

Figure 4. The stress nephogram of expansion joint.

(2) When the horizontal displacement is $9 \mathrm{~mm}$, the stress nephogram is shown in figure 5.

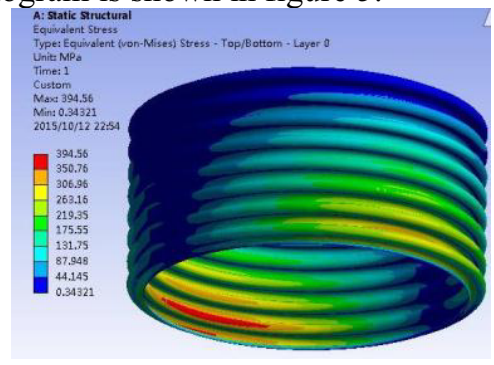

Figure 5. The stress nephogram of expansion joint.

Stress simulation results show that when the expansion joint stress deformed, the highest stress position is on the end of corrugated pipe where is connecting with the pipe. The simulation result is consistent with the deformed position. When the horizontal displacement is $4 \mathrm{~mm}$, the maximum stress is $175.56 \mathrm{Mpa}{ }^{[5]}$; When the horizontal displacement is 9 $\mathrm{mm}$, the maximum stress is up to $394.56 \mathrm{Mpa}$, almost excesses material yield limit ${ }^{[6]}$. 


\section{Analysis and discussion}

1) The metallography analysis of the MSR expansion joint shows that the expansion joint is made of $304 \mathrm{~L}$ stainless steel, $\mathrm{C}$ element content of the expansion joint is beyond the specified value in the ASTM A240[7]. The hardness values of inside and outside parts of expansion joint are also significantly higher than the specified value in ASTM A240. High hardness makes the material's brittleness increase, toughness decrease. When materials under external force beyond yield limit, it will prone to cracking.

2)When the expansion joint is under the force, the highest stress position is on the end of the corrugated pipe where is connecting with the pipe. The simulation results are in conformity with the deformation position. Expansion joint folding deformation position locates in the maximum horizontal displacement. Macro and micro analysis shows, the welding ends and corrugated pipe has no abnormalities, those exclude the possibility that gas first came into the expansion joint interlayer. When the interlayer of expansion joint has water, the expansion joint has condition to deform. Based on the above evidence, this paper thinks that the innermost layer of expansion joint in maximize deformation first occurs to crack.

\section{Conclusions}

For the MSR expansion joint folding deformation problem, this paper analysis through the on-site survey, displacement measurement analysis, laboratory analysis and the stress calculation analysis, etc. The analysis results show the horizontal displacement allowance is small leading the hydrophobic expansion joint has poor adaptation. When the expansion joint is installed, the horizontal displacement is beyond standard. After the unit running, the horizontal displacement beyond design standards, and the expansion joint material hardness values exceed standard, finally leading to the expansion joint fatigue life greatly reduced.

After operation, the expansion joint of MSR appearing thermal expanding displacement makes expansion joint under the force. The expansion joint innermost layer 1 where connecting with pipe has forced the maximum stress began to cracking. When the unit start-up, the working medium at low temperature coming into the interlayer. As the unit power rising, the working medium which come into layers became vaporization with temperature rising, affecting internal pressure of the expansion joint interlayer, making the inside and outside expansion joint has different pressure, and finally leading to folding deformation [8].

Through comprehensive analysis, determined the root cause of the hydrophobic expansion joint deformation is: the expansion joint adaption is poor which is easy to lead the installation has big deviation, and the material hardness values exceed the standard. The contributing cause of the problem is: the material $\mathrm{C}$ element content beyond the standard.

\section{Improvement measures}

According to the nuclear power plant hydrophobic expansion joint deformation problem, making the following improvements:

1) Given the expansion joint has quality defects, whose displacement has small allowance and installation precision is difficult to control, suggesting that choosing bigger displacement allowance expansion joint, and waiting right time to replace it.

2) Before the expansion joint replacement has been finished, evaluating the expansion joint of MSR system. According to the evaluation results, arrange the reasonable replacement order.

3) Update version of installation procedures, the expansion joint of the original horizontal displacement and radial displacement measurement, ensuring that the expansion joint displacement allowance is enough.

\section{References}

1. Guangdong nuclear power training center. Devices and Systems of 900MW PWR [M]. Beijing: Atomic Energy Press.

2. Chen Lisu and so on. The application and design manufacturing characteristics of nuclear level's expansion joint. Pipeline technique and equipment. 2012(1)

3. Dong Tingkai. The failure reasons of expansion joint [J]. Shandong metallurgical. 2006, 28(1): 67-68.

4. Hou Jinghong. Handling of over-proof horizontal displacement of the expansion joint for the re-heater in nuclear power station[J]. Power Station Auxiliary Equipment. 2012, 33(2)

5. Zhao Cheng long .Engineering thermodynamics [M], Nanjing aviation professional teaching materials published editorial group,1984, 2.

6. Zhao Jinxing and so on. External pressure type corrugated expansion joint failure case analysis[J], Pipeline technology and equipment, 2002(8)

7. Stainless steel and heat resistant steel general requirements for rolling steel plate, sheet and strip ASTM SA-480/SA-480M.

8. General technical requirements of metal bellows expansion joint. GB/T 12777- 2008.Bejing: National Quality Technical Supervise Department. 2008, 6 